\title{
Pulmonary function is implicated in the prognosis of metastatic non-small cell lung cancer but not in extended disease small cell lung cancer
}

\author{
Suk-young Lee ${ }^{1}$, Yoon Ji Choi ${ }^{1}$, Jae Hong Seo ${ }^{1}$, Sung Yong Lee ${ }^{2}$, Jung Sun Kim ${ }^{1}$, Eun Joo Kang ${ }^{1}$ \\ ${ }^{1}$ Division of Oncology/Hematology, ${ }^{2}$ Division of Pulmonology, Department of Internal Medicine, College of Medicine, Korea University, Seoul \\ 08308, Republic of Korea \\ Contributions: (I) Conception and design: EJ Kang, SY Lee; (II) Administrative support: EJ Kang, JH Seo; (III) Provision of study materials or \\ patients: SY Lee, YJ Choi, SY Lee, JS Kim, EJ Kang; (IV) Collection and assembly of data: SY Lee, EJ Kang; (V) Data analysis and interpretation: \\ SY Lee; (VI) Manuscript writing: All authors; (VII) Final approval of manuscript: All authors. \\ Correspondence to: Eun Joo Kang. Division of Oncology/Hematology, Department of Internal Medicine, College of Medicine, Korea University, 148 \\ Gurodong-ro, Guro-gu, Seoul 08308, Republic of Korea. Email: kkangju11@naver.com.
}

Background: The impacts of pulmonary function in patients with metastatic non-small cell lung cancer (NSCLC) and extended disease stage small cell lung cancer (SCLC-ED) treated with palliative chemotherapy remain to still be determined.

Methods: Results of spirometry performed in 449 patients with either stage IV NSCLC ( $\mathrm{n}=313)$ or SCLCED ( $n=136)$ at diagnosis were reviewed retrospectively. Overall survival (OS) was estimated using the Kaplan-Meier method and compared via a log-rank test. Multivariate analysis was performed using a Cox proportional hazards regression model.

Results: The presence of chronic obstructive pulmonary disease (COPD) was not a risk factor for OS in either NSCLC or SCLC. However, NSCLC patients with COPD with a forced expiratory volume in one second (FEV1) value of less than $80 \%$ predicted were associated with a worse OS in both univariate and multivariate analyses [hazard ratio (HR): 1.43; 95\% confidence interval (CI): 1.04-1.97; P=0.03]. Intriguingly, only the OS of NSCLC patients treated with chemotherapeutic agents was affected by the airflow limitation FEV1 value of less than $80 \%$ predicted $(\mathrm{P}=0.02)$. Patients with an $\mathrm{FEV} 1$ value of less than $80 \%$ predicted treated with targeted agents were not associated with OS $(\mathrm{P}=0.24)$. On the other hand, NSCLC patients with COPD were significantly linked to the occurrence of pulmonary complications during palliative therapy $(\mathrm{P}=0.01)$ but not associated with death resulting from pulmonary complications $(\mathrm{P}=0.22)$.

Conclusions: Careful attention is required when chemotherapeutic agents are administered to patients with metastatic NSCLC with accompanying COPD with a FEV1 value of less than $80 \%$ predicted.

Keywords: Advanced lung cancer; obstructive lung disease; lung function; prognostic indicator

Submitted May 02, 2019. Accepted for publication Oct 21, 2019.

doi: $10.21037 /$ jtd.2019.10.77

View this article at: http://dx.doi.org/10.21037/jtd.2019.10.77

\section{Introduction}

Lung cancer (LC) is the leading cause of cancer mortality worldwide (1). Existing comorbidities are crucial factors to be considered in planning the treatment for patients with non-small cell lung cancer (NSCLC). Because chronic obstructive pulmonary disease (COPD) is one of the most commonly known comorbidities in LC patients, the prognosis for patients with NSCLC and comorbid COPD has been examined in several previous studies (2-9). Some of these studies reported a worse prognosis in NSCLC patients with COPD versus in those without COPD $(2-5,9)$, while other investigations showed the comparable survival 
rates between the two groups (6-8). The inconsistent results of previous studies could be partly explained by the heterogeneity of patients evaluated in terms of American Joint Commission on Cancer staging status (10), histology, and treatment modalities. A recent meta-analysis examining the prognosis of LC patients with COPD revealed a poorer overall survival (OS) for those patients with concomitant COPD than for those without COPD. The same result was shown in patients with LC presenting with emphysema versus without. However, while the presence of COPD in patients with LC was associated with a worse OS in the subgroup analysis focusing on stages I to II patients, no conclusion has been established regarding patients with advanced LC (11).

In addition, there exists a lack of data to refer to when choosing therapeutic agents for LC patients with COPD. Along with extensive research on the molecular mechanisms of the tumorigenesis of LC, many targeted agents have been developed so that a variety of treatment agents including conventional chemotherapeutic agents can be incorporated into individualized therapy strategies at present (12-16). Different toxicity profiles between targeted agents and conventional chemotherapeutic agents made us think there was a need to address the impact of COPD on the prognosis of patients with NSCLC according to the different therapeutic agents.

In patients with extended disease stage small cell lung cancer (SCLC-ED), there is no option other than the systemic administration of chemotherapeutic agents in the front-line treatment. Several studies have reported an association between the pulmonary function and prognosis of patients with SCLC. Cho et al. reported that the low forced expiratory volume in one second (FEV1)/forced vital capacity (FVC) ratio was a poor prognostic factor for patients with limited disease stage (SCLC-LD) (17). Kang et al. separately noted that low FEV1 was associated with shorter survival in patients with SCLC, with sub-group analysis showing that low FEV1 was also a poor prognostic factor for patients with SCLC-ED (18). However, a different recent study reported that COPD had no impact on the OS of patients with SCLC who received first-line chemotherapy (19). Although these discordant results could be attributed to several reasons, the heterogeneous clinical characteristics of the included patients could be one of main reasons for such. For the lack of studies involving an analysis of the relationship between COPD and the prognosis of patients with SCLC-ED who received palliative chemotherapy, attempt to examine effects of
COPD on the treatment outcomes of patients with SCLCED who received palliative chemotherapy was made in this study. In addition, the impacts of COPD on prognosis for patients with stage IV NSCLC as well as on prognosis of those patients according to respective therapeutic agents used in treatment were investigated.

\section{Methods}

A total of 449 patients diagnosed with advanced LC between May 2002 and May 2017 at the Korea University medical center were retrospectively analyzed. One hundred and thirty-six patients were diagnosed with SCLC-ED, while 313 patients had metastatic NSCLC. Both SCLCED and metastatic NSCLC patients with available spirometry data at diagnosis were included in this study. Patients with coexisting asthma or a medical history of asthma were excluded. Spirometric and diffusing capacity for carbon monoxide (DLCO) norms in Korean pulmonary function test guideline (2016) were applied (20). This study was carried out according to the principles in the 1964 Declaration of Helsinki, and an ethical committee approved the study protocol (2018GR0219). Because this study was performed in retrospective manner informed consent was waived.

LC diagnosis and histological classification were established by well-experienced pathologists using tumor tissue acquired via biopsy. COPD was defined in accordance with the Global Initiative for Chronic Obstructive Lung Disease (GOLD) guidelines as a post-bronchodilator FEV1/FVC ratio of less than 0.7 with spirometry. The severity of airflow limitation was categorized into four stages considering the percentage of predicted postbronchodilator FEV1 according to the GOLD classification, as follows: GOLD 1: mild (FEV1 $\geq 80 \%$ predicted); GOLD 2: moderate $(50 \% \leq \mathrm{FEV} 1<80 \%$ predicted); GOLD 3: severe $(30 \% \leq \mathrm{FEV} 1<50 \%$ predicted); and GOLD 4 : very severe (FEV1 $<30 \%$ predicted) (21). The response to chemotherapy was evaluated using computed tomography (CT) according to the criteria of the Response Evaluation Criteria in Solid Tumours (RECIST) ver.1.1 (22). Complete response (CR) is defined as the absence of all target lesions with any pathologic lymph nodes smaller than $10 \mathrm{~mm}$ on the short axis. A decrease of $30 \%$ or more in the sum of diameters of target lesions from the baseline sum diameters is defined as partial response (PR). Progressive disease (PD) is defined as an increase of $20 \%$ or more in the sum of diameters of target lesions from the smallest sum of 
the study with an absolute increase in diameter of $5 \mathrm{~mm}$ with or without the appearance of new lesions. Stable disease (SD) is any condition that did not meet the criteria described above. Body mass index (BMI) was calculated as the individual's body mass $(\mathrm{kg})$ divided by the square of height $\left(\mathrm{m}^{2}\right)$. A BMI $\geq 25.0 \mathrm{~kg} / \mathrm{m}^{2}$ was defined as obese based on Asia-Pacific criteria (23). Diabetes mellitus (DM), hypertension, pulmonary tuberculosis, and cardiovascular disease were investigated as comorbidities through a review of patient medical records.

The clinical parameters evaluated to determine an association with OS were age $\geq 70$ years, sex (male $v s$. female), East Cooperative Oncology Group (ECOG) performance status (PS) (0-1 vs. 2-3) (24), a BMI $\geq 25 \mathrm{~kg} / \mathrm{m}^{2}$, smoking history (current and former smokers $v s$. never smokers), tumor histology (adenocarcinoma vs. squamous cell carcinoma and others), presence of COPD, site of disease, presence of DM, FEV $1<80 \%$ predicted, and a DLCO $<60 \%(18,25,26)$. Pulmonary complications examined to see an association with COPD were pneumonia, COPD exacerbation, interstitial lung disease (ILD), radiation pneumonitis, pulmonary embolism, and pneumothorax. Pneumonia was diagnosed by confirming acute infection of pulmonary parenchyma. COPD exacerbation was defined as a sudden worsening of COPD symptoms which requires additional medications. ILD was diagnosed when patients had history of exposure to causative antineoplastic agents, respiratory symptoms such as fever and dyspnea, and CT findings suggestive ILD after excluding other conditions causing pulmonary opacities. Radiation pneumonitis was diagnosed if opacities are detected alongside the irradiated area with chest CT in patients who had had previous radiation therapy. Pulmonary embolism was diagnosed with elevated d-dimer level and a CT pulmonary angiography finding.

Initially recorded continuous laboratory variables were dichotomized for ease of statistical analysis. Clinical characteristics were compared between the COPD and non-COPD groups using the chi-square test. Results of spirometry were compared using the Student's $t$-test. OS was defined as the period from the first date of firstline chemotherapy to the date of any cause of death. The Kaplan-Meier method was employed to estimate OS and the log-rank test was used to compare OS according to the clinical parameters. Multivariate analysis was performed using the Cox proportional hazards regression model to address independently associated significant prognostic factors for OS. ECOG PS and smoking history were not included for analyses because of concern on inaccuracy in the aspect of retrospective data. Stepwise model selection algorithm was used for the multivariate analysis in the stage IV NSLCL group. Results were described as the hazard ratio (HR) and $95 \%$ confidence interval (CI). A $\mathrm{P}$ value $<0.05$ was considered statistically significant. Associations between COPD and the occurrence of pulmonary complications during chemotherapy or death due to pulmonary complications were examined using the chi-square test. An association between severity of airflow limitation and tumor histology (adenocarcinoma and other histological subtypes) was also determined using chi-square test. Statistical analysis was performed using the Statistical Package for the Social Sciences version 20 for Windows (IBM Corp., Armonk, NY, USA).

\section{Results}

\section{Patients with SCLC-ED}

The characteristics of the 136 patients with SCLC-ED who performed a pulmonary function test at the time of diagnosis and who received palliative chemotherapy are summarized in Table 1. Patients were further classified into the two groups of non-COPD ( $n=62)$ and COPD $(n=74)$. Baseline characteristics, including age, sex, history of smoking, site of disease, and ECOG PS status did not differ significantly between the two groups. The results of spirometry were also comparable between the two groups except for FEV1/ FVC $(\mathrm{P}<0.01)$ and FEV1 $(\mathrm{P}<0.01)$. The median age of all patients was 69 years (range, $45-83$ years). One hundred and eleven $(81.6 \%)$ patients were male and $119(87.5 \%)$ patients were current or former smokers. Additionally, 94 (69.1\%) patients received palliative chemotherapy only, while $42(30.9 \%)$ patients received palliative chemotherapy and palliative radiotherapy irradiated to the bone, lung, or brain. One hundred and thirty-three $(97.8 \%)$ patients received platinum doublets.

The median OS was 8.7 months (95\% CI: 8.0-9.4 months) with a median follow-up of 73.4 months. The median OS rates between the non-COPD group (8.6 months, $95 \%$ CI: $6.3-11.0)$ and the COPD group (8.7 months, $95 \% \mathrm{CI}$ : 8.1-9.3 months) were not significantly different $(\mathrm{P}=0.80)$ in patients with SCLC-ED (Figure 1A). Separately, the median OS values for the non-COPD and COPD groups according to the severity defined per the GOLD criteria were also not significantly different $(\mathrm{P}=0.83)$. Next, we investigated the prognostic factors for $\mathrm{OS}$ in patients with 
Table 1 Characteristics of patients with lung cancer $(n=449)$

\begin{tabular}{|c|c|c|c|c|c|c|}
\hline Characteristics & \multicolumn{3}{|c|}{ SCLC-ED } & \multicolumn{3}{|c|}{ Stage IV NSCLC } \\
\hline Age, n (\%) & & & 0.29 & & & $<0.01$ \\
\hline$\geq 70$ years & $39(52.7)$ & $27(43.5)$ & & $74(54.0)$ & $62(35.2)$ & \\
\hline$<70$ years & $35(47.3)$ & $35(56.5)$ & & $63(46.0)$ & $114(64.8)$ & \\
\hline Male & $62(83.8)$ & $49(79.0)$ & & $115(83.9)$ & $97(55.1)$ & \\
\hline Female & $12(16.2)$ & $13(21.0)$ & & $22(16.1)$ & $79(44.9)$ & \\
\hline Body mass index, $\mathrm{n}(\%)$ & & & 0.05 & & & 0.02 \\
\hline$\geq 25.0 \mathrm{~kg} / \mathrm{m}^{2}$ & $11(14.9)$ & $18(29.0)$ & & 25 (18.2) & $52(29.5)$ & \\
\hline Yes & $9(12.2)$ & $18(29.0)$ & & $30(21.9)$ & $37(21.0)$ & \\
\hline No & $65(87.8)$ & $44(71.0)$ & & $107(78.1)$ & $139(79.0)$ & \\
\hline Smoking history, n (\%) & & & 0.24 & & & $<0.01$ \\
\hline Current and former & $67(90.5)$ & $52(83.9)$ & & $108(78.8)$ & $97(55.1)$ & \\
\hline Never & $7(9.5)$ & $10(16.1)$ & & $29(21.2)$ & $79(44.9)$ & \\
\hline ECOG PS, n (\%) & & & 0.25 & & & $<0.01$ \\
\hline $0-1$ & $65(87.8)$ & $50(80.6)$ & & $108(78.8)$ & $158(89.8)$ & \\
\hline $2-3$ & $9(12.2)$ & $12(19.4)$ & & $29(21.2)$ & $18(10.2)$ & \\
\hline 2 & $45(60.8)$ & - & & $78(56.9)$ & - & \\
\hline 3 & $10(13.5)$ & - & & $22(16.1)$ & - & \\
\hline 4 & 0 & - & & $2(1.5)$ & - & \\
\hline FEV1/FVC (mean \pm SD) (\%) & $58.11 \pm 7.89$ & $84.85 \pm 61.80$ & $<0.01$ & $60.42 \pm 8.25$ & $78.37 \pm 7.08$ & $<0.01$ \\
\hline FEV1 (mean \pm SD) $(\%)$ & $68.97 \pm 18.99$ & $79.42 \pm 22.15$ & $<0.01$ & $66.64 \pm 17.97$ & $85.93 \pm 19.23$ & $<0.01$ \\
\hline$\geq 80, \mathrm{n}(\%)$ & $19(25.7)$ & $32(51.6)$ & $<0.01$ & $39(28.5)$ & $114(64.8)$ & $<0.01$ \\
\hline$<80, \mathrm{n}(\%)$ & $55(74.3)$ & $30(48.4)$ & & $98(71.5)$ & $62(35.2)$ & \\
\hline DLCO, n (\%) & $n=59$ & $\mathrm{n}=45$ & 0.81 & $n=109$ & $n=133$ & 0.28 \\
\hline$\geq 60$ & $42(71.2)$ & $33(73.3)$ & & $76(69.7)$ & $101(75.9)$ & \\
\hline$<60$ & $17(28.8)$ & $12(26.7)$ & & $33(30.3)$ & $32(24.1)$ & \\
\hline
\end{tabular}

Table 1 (continued) 
Table 1 (continued)

\begin{tabular}{|c|c|c|c|c|c|c|}
\hline Characteristics & \multicolumn{3}{|c|}{ SCLC-ED } & \multicolumn{3}{|c|}{ Stage IV NSCLC } \\
\hline Histology, n (\%) & & & - & & & - \\
\hline Adenocarcinoma & - & - & & $94(68.6)$ & $152(86.4)$ & \\
\hline Squamous cell carcinoma & - & - & & $34(24.8)$ & $17(9.7)$ & \\
\hline Others & - & - & & $7(5.1)$ & $6(3.4)$ & \\
\hline \multicolumn{7}{|l|}{ Treatment agents (n, \%) } \\
\hline Targeted agent & & & - & & & - \\
\hline Erlotinib & - & - & & $2(1.5)$ & $7(4.0)$ & \\
\hline Chemotherapeutic agent & & & - & & & - \\
\hline Pemetrexed + cisplatin & - & - & & $31(22.6)$ & $59(33.5)$ & \\
\hline Gemcitabine + carboplatin & - & - & & $51(37.2)$ & $28(15.9)$ & \\
\hline Gemcitabine + cisplatin & - & - & & $12(8.8)$ & $17(9.7)$ & \\
\hline Pulmonary complication, $\mathrm{n}(\%)$ & - & - & - & $41(29.9)$ & $31(17.6)$ & 0.01 \\
\hline Pneumonia & - & - & & $34(24.8)$ & $30(17)$ & \\
\hline COPD exacerbation & - & - & & $9(6.6)$ & $1(0.6)$ & \\
\hline ILD & - & - & & $1(0.7)$ & $1(0.6)$ & \\
\hline
\end{tabular}

COPD, chronic obstructive pulmonary disease; DLCO, diffusing capacity for carbon monoxide; ECOG, East Cooperative Oncology Group; FEV1, forced expiratory volume in 1 second; FVC, forced vital capacity; GOLD, Global Initiative for Chronic Obstructive Lung Disease; ILD, interstitial lung disease; NSCLC, non-small cell lung cancer; PS, performance status; SCLC-ED, extended disease stage small cell lung cancer; SD, standard deviation.

SCLC-ED. Univariate analysis showed FEV1 and DLCO did not predict survival for this patient group. Among the clinical factors, higher age $\geq 70$ years, presence of $\mathrm{DM}$, poor ECOG PS $(\geq 2)$, non-responder to first-line chemotherapy $(\mathrm{SD}+\mathrm{PD})$, and short duration of first-line chemotherapy (less than 4 cycles) significantly predicted poor prognosis $(\mathrm{P}<0.05)$. Multivariate analysis showed that higher age (HR: 2.01; 95\% CI: 1.39-2.91; $\mathrm{P}<0.01$ ), presence of DM (HR: 1.87; 95\% CI: $1.17-2.99 ; \mathrm{P}<0.01)$, non-responder to firstline chemotherapy (HR: 1.86; 95\% CI: 1.19-2.92; $\mathrm{P}<0.01$ ), and shorter duration of chemotherapy (HR: $0.22 ; 95 \%$ CI: $0.14-0.36$; $\mathrm{P}<0.01)$ were independent prognostic factors for
OS (Table 2).

\section{Patients with stage IV NSCLC}

The characteristics of 313 patients diagnosed with stage IV NSCLC with available spirometry results are summarized in Table 1. The characteristics of patients in the COPD group $(n=137)$ and the non-COPD group $(n=176)$ were compared. A larger proportion of patients were younger than 70 years in the non-COPD group. The ratio of males to females was 5.2:1 in the COPD group. The ECOG PS of most patients was less than two in both groups. As expected, patients with 

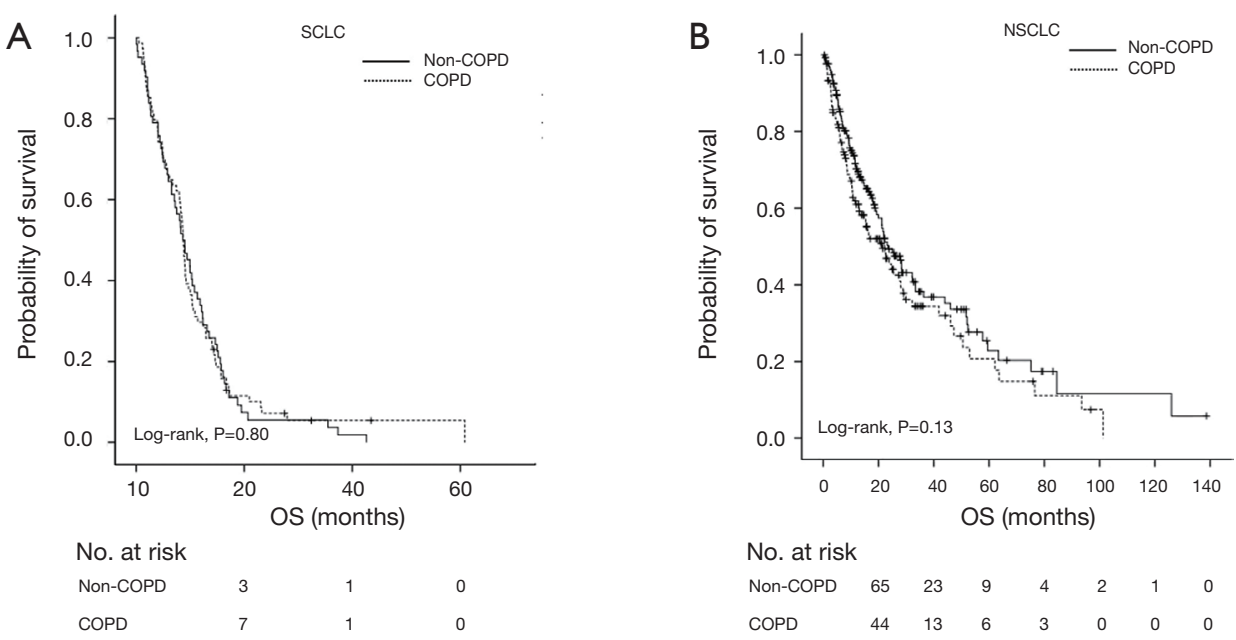

Figure 1 OS did not differ between the COPD and non-COPD groups in patients with (A) SCLC and (B) NSCLC. OS, overall survival; COPD, chronic obstructive pulmonary disease; SCLC, small cell lung cancer; NSCLC, non-small cell lung cancer.

Table 2 Prognostic factors for OS in patients with SCLC-ED ( $\mathrm{n}=136)$

\begin{tabular}{|c|c|c|c|c|}
\hline Variables & Death/total, $\mathrm{n}$ & $\begin{array}{c}\text { Univariate } \\
\mathrm{P} \text { value }\end{array}$ & \multicolumn{2}{|c|}{ Multivariate } \\
\hline Age (<70 vs. $\geq 70)$ & $68 / 70$ vs. $63 / 66$ & $<0.01$ & $2.01(1.39-2.91)$ & $<0.01$ \\
\hline Sex (male vs. female) & $107 / 111$ vs. $24 / 25$ & 0.62 & - & - \\
\hline Diabetes mellitus (no vs. yes) & $105 / 109$ vs. $26 / 27$ & 0.04 & $1.87(1.17-2.99)$ & $<0.01$ \\
\hline Smoking history (never vs. current and former) & $16 / 17$ vs. $115 / 119$ & 0.51 & - & - \\
\hline Body mass index (<25 vs. $\left.\geq 25 \mathrm{~kg} / \mathrm{m}^{2}\right)$ & $103 / 107$ vs. $28 / 29$ & 0.18 & - & - \\
\hline Site of disease (central vs. peripheral) & $128 / 132$ vs. $3 / 4$ & 0.98 & - & - \\
\hline Response to $1 \mathrm{~L}$ chemotherapy (CR + PR vs. SD + PD) & $78 / 83$ vs. $53 / 53$ & $<0.01$ & $1.86(1.19-2.92)$ & $<0.01$ \\
\hline Non-COPD and GOLD 1 vs. GOLD 2-4 & $79 / 81$ vs. $52 / 55$ & 0.71 & - & - \\
\hline DLCO (<60\% vs. $\geq 60 \%)$ & $28 / 29$ vs. $72 / 75$ & 0.49 & - & - \\
\hline
\end{tabular}

SCLC-ED, extended disease stage small cell lung cancer; Cl, confidence interval; ECOG, East Cooperative Oncology Group; PS, performance status; CR, complete response; PR, partial response; SD, stable disease; PD, progressive disease; GOLD, Global Initiative for Chronic Obstructive Lung Disease; HR, hazard ratio; L, line; OS, overall survival; COPD, chronic obstructive pulmonary disease; DLCO, diffusing capacity for carbon monoxide.

a smoking history composed a larger proportion of both groups. Adenocarcinoma was the most frequently diagnosed histology type in both groups. The results of spirometry for FEV1/FVC and FEV1 were significantly different between the COPD and non-COPD groups $(\mathrm{P}<0.01)$. Pemetrexed and cisplatin combination chemotherapy and combined gemcitabine and carboplatin chemotherapy were the most frequently applied regimens to patients in the non-COPD and the COPD group, respectively. A total of 72 patients with NSCLC experienced pulmonary complications 
Table 3 Prognostic factors for OS in patients with stage IV NSCLC $(\mathrm{n}=313)$

\begin{tabular}{|c|c|c|c|c|}
\hline Variables & Death/total, n & $\begin{array}{c}\text { Univariate } \\
\mathrm{P} \text { value }\end{array}$ & \multicolumn{2}{|c|}{ Multivariate } \\
\hline Age (<70 vs. $\geq 70)$ & $105 / 177$ vs. $70 / 136$ & 0.38 & - & - \\
\hline Sex (male vs. female) & $123 / 212$ vs. $52 / 101$ & $<0.01$ & - & - \\
\hline Co-morbidities (no vs. yes) & $59 / 105$ vs. $115 / 207$ & 0.34 & - & - \\
\hline Smoking history (never vs. current and former) & $56 / 102$ vs. $117 / 205$ & 0.01 & - & - \\
\hline Body mass index (<25 vs. $\left.\geq 25 \mathrm{~kg} / \mathrm{m}^{2}\right)$ & $133 / 236$ vs. $42 / 77$ & 0.19 & - & - \\
\hline Site of disease (central vs. peripheral) & $75 / 128$ vs. $100 / 185$ & 0.12 & - & - \\
\hline Histology (adenocarcinoma vs. other histology) & $134 / 246$ vs. $41 / 67$ & $<0.01$ & $1.69(1.17-2.43)$ & $<0.01$ \\
\hline Non-COPD and GOLD 1 vs. GOLD 2-4 & $113 / 211$ vs. $62 / 102$ & $<0.01$ & $1.43(1.04-1.97)$ & 0.03 \\
\hline DLCO (<60\% vs. $\geq 60 \%)$ & $20 / 65$ vs. $43 / 177$ & 0.01 & - & - \\
\hline
\end{tabular}

OS, Overall survival; NSCLC, Non-small cell lung cancer; HR, Hazard ratio; Cl, Confidence interval; ECOG, East Cooperative Oncology Group; PS, Performance status; COPD, Chronic obstructive pulmonary disease; GOLD, Global Initiative for Chronic Obstructive Lung Disease; DLCO, diffusing capacity for carbon monoxide.

during the course of treatment. Pneumonia was the most frequently recorded pulmonary complication in both groups. COPD exacerbation was the second most common pulmonary complication in the COPD group. One patient whose FEV1/FVC ratio had been within the normal range at the time of diagnosis developed COPD and COPD exacerbation during the course of chemotherapy.

With a median follow-up of 56.6 months, the median OS was 22.6 months (95\% CI: 17.8-27.3 months). The median OS between the two groups was not significantly different in patients with NSCLC (non-COPD group: 23.5 months, 95\% CI: 18.1-28.8 months; COPD group: 21.3 months, 95\% CI: 13.7-28.8 months; $\mathrm{P}=0.13$ ) (Figure 1B). However, the severity of COPD airflow limitation defined according to the GOLD classification was found to affect on prognosis in terms of OS. The OS for patients with FEV1/FVC $\geq 0.7$ and those classified GOLD 1 was significantly different from that of patients classified GOLD 2-4 $(\mathrm{P}<0.01)$. A univariate analysis showed patients with a DLCO less than $60 \%$ also had significantly worse OS $(\mathrm{P}=0.01)$. Univariate analyses showed that clinical parameters significantly associated with OS were sex, ECOG PS (0-1 vs. 2-3), current or past history of smoking, histology type (adenocarcinoma $v s$. other histology types), and treatment agent $(\mathrm{P}<0.05)$. Multivariate analysis showed NSCLC patients with FEV1/FVC $\geq 0.7$ and those classified GOLD 1 (HR: 1.43; 95\% CI: 1.04-1.97; $\mathrm{P}=0.03$ ), those harboring tumors classified as adenocarcinoma (HR: 1.69; 95\% CI: $1.17-2.43 ; \mathrm{P}<0.01)$, and those treated with targeted agents (HR: 2.70 ; 95\% CI: 1.55-4.70; $\mathrm{P}<0.01$ ) had favorable OS (Table 3). Larger numbers in patients with adenocarcinoma (178 of 246 patients) were classified GOLD 1 or had no airflow limitation $(\mathrm{P}<0.01)$.

Although stage IV NSCLC patients with COPD had pulmonary complications more frequently than those without COPD during the course of chemotherapy $(\mathrm{P}=0.01)$, deaths resulting from pulmonary complications were comparable between the two groups $(\mathrm{P}=0.22)$. Therapeutic agents were also found to exert an effect upon the prognosis of stage IV NSCLC patients with COPD. Patients treated with targeted agents showed no significant difference in OS according to airflow limitation severity (patients with FEV1/FVC $\geq 0.7$ and those GOLD 1 vs. patients GOLD 2-4, $\mathrm{P}=0.24$ ) (Figure $2 A$ ). Meanwhile, the OS of stage IV NSCLC patients treated with conventional chemotherapeutic agents was significantly different according to the severity of airflow limitation. Stage IV NSCLC patients classified GOLD 1 showed a significantly 

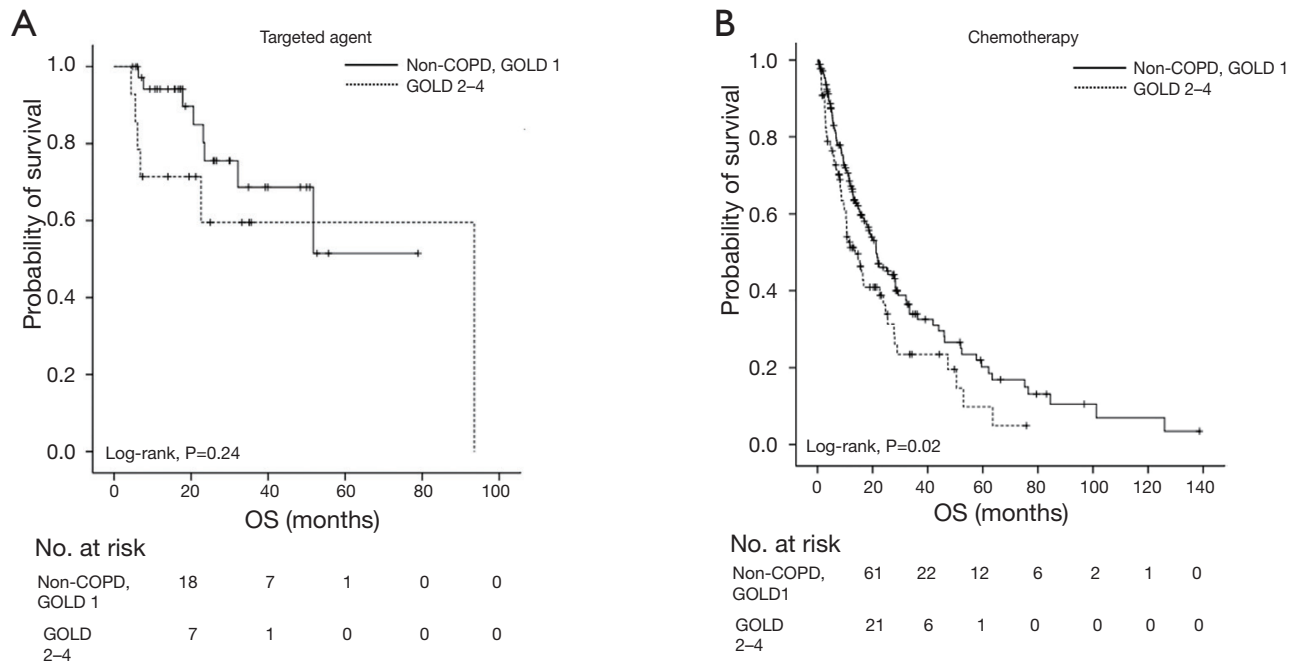

Figure 2 The OS of patients with stage IV NSCLC. (A) The OS of patients treated with targeted agents was not affected by the severity of airflow limitation; (B) the OS of patients treated with conventional chemotherapy was significantly different according to the severity of airflow limitation. OS, overall survival; NSCLC, non-small cell lung cancer; GOLD, chronic obstructive lung disease.

better OS compared to those classified GOLD 2-4 upon chemotherapy treatment $(\mathrm{P}=0.02)$ (Figure $2 B)$.

\section{Discussion}

Although several previous studies have investigated the impacts of COPD on the prognosis of patients with LC, no consistent results have been reported to date. In order to exclude confusing factors attributed to the heterogeneous characteristics of patients, only patients with advanced LC, specifically stage IV NSCLC and SCLC-ED, were analyzed in this study. The results of our analyses indicate that COPD is not significantly associated with the survival of patients with metastatic NSCLC or SCLC-ED.

The results of previous studies that investigated the effects of pulmonary function on survival of SCLC patients suggested there was an adverse prognosis for SCLC patients with airflow limitation, though inconsistent indicators for airway limitation such as FEV1 or FEV1/ FVC were used $(17,18)$. As a matter of fact, treatment is recommended to be tailored according to the results of preoperative pulmonary function tests in patients with LC who are required to undergo surgical resection for the risk resulting from lung resection (27). It is also known that the adoption of treatment modalities is influenced by coexisting COPD in NSCLC patients. A previous study reported that NSCLC patients with COPD underwent surgery and chemotherapy less frequently than those without COPD despite the lack of data regarding the adverse effects of COPD on the prognosis of patients receiving palliative chemotherapy (28). One recent investigation that addressed the impact of COPD on the OS of patients with SCLC receiving chemotherapy reported coexisting COPD had no association with survival in those patients (19). Our study, which involved only SCLC-ED patients, also showed no association between COPD or severity of air flow limitation and prognosis in terms of OS, consistent to results of the previous study (19), suggesting that treatment with chemotherapy could be considered as a prior treatment option in patients with SCLC-ED.

On the other hand, although the presence of COPD itself was not a poor prognostic factor in patients with metastatic NSCLC, the severity of airflow limitation exerted an effect on the survival of those patients in our study. Intriguingly, survival did not differ according to the severity of airflow limitation in patients who received targeted agents such as tyrosine kinase inhibitors; meanwhile, the OS of patients given conventional chemotherapeutic agents was affected by the COPD airflow limitation severity. Whether the observed difference in survival was attributable to death resulting from pulmonary complications was examined, but the proportion of patients who died due to pulmonary complications was not significantly different between the COPD and non-COPD groups in NSCLC patients given chemotherapy ( $\mathrm{P}=0.14$, data not shown), suggesting compromised lung function is not the only medical problem 
to be taken care of during treatment with chemotherapy. A previous study on treatment outcomes for NSCLC patients with COPD who received chemotherapy reported positive effects of chemotherapy compared with supportive care $(8,29)$, but results from our study indicated that COPD classified as GOLD 2 or worse had an unfavorable impact on treatment outcomes of chemotherapy. A previous study reported that the OS of patients with NSCLC who have an effective host DNA repair system is significantly worse than that of NSCLC patients with a suboptimal DNA repair system in conjunction with chemotherapy treatment (30). Therefore, the different individual repair capabilities of DNA damage caused by oxidative stress during the development of COPD (31) might have contributed to the differences in OS according to the severity of airway limitation in patients with NSCLC treated with chemotherapy. In addition, in our study patients with COPD were shown to be associated with many other comorbidities such as DM or cardiovascular disease $(\mathrm{P}=0.02)$. This could lead to complications brought on by chemotherapy, resulting in a discontinuation of treatment, which consequently might affect the prognosis of patients receiving chemotherapy. Taken together, because a poor treatment outcome is anticipated in NSCLC patients with a FEV1 value of less than $80 \%$ predicted, especially when these people are treated with conventional chemotherapeutic agents, careful attention should be paid to such individuals when they are given chemotherapy.

A low DLCO has been reported to be associated with poor survival in patients with LC including NSCLC and SCLC $(25,26)$. This was also the case in patients with SCLC-ED and stage IV NSCLC in univariate analyses, but the multivariate analysis has not been performed in this study because results of DLCO were available in only parts of patients $(n=242)$. For lack of data on relationship between DLCO and prognosis of advanced LC, further investigation with larger number of patients is necessary. Adenocarcinoma histology type was one of the independent favorable prognostic factors, a finding consistent with previous studies (32). On the other hand, a previous study that addressed the impact of COPD on the prognosis of patients with LC according to histology subtype reported that the coexistence of COPD as well as its severity were not significantly associated with the prognosis for patients harboring the adenocarcinoma subtype (33). In our study, the OS of NSCLC patients with adenocarcinoma did not differ significantly according to the presence of COPD $(\mathrm{P}=0.46$, data not shown); meanwhile, the severity of airflow limitation affected the prognosis of those patients (FEV1 $\leq 80 \%$ predicted; $\mathrm{P}=0.03$, data not shown). Intriguingly, larger numbers in patients harboring adenocarcinoma were found to be classified GOLD 1 or had no COPD, and this might have been one of explanations for better prognosis of those patients comparing to NSCLC patients with histology subtypes other than adenocarcinoma.

The results in this study revealed that there was no impact of COPD on the OS of SCLC-ED patients who received palliative chemotherapy, while poor prognosis in stage IV NSCLC patients with GOLD 2-4 was observed, suggesting the presence of COPD should not be a contraindication for palliative chemotherapy in patients with SCLC-ED or NSCLC classified GOLD 1. Although efforts to overcome limitations of previous studies attributing to heterogeneity of patients characteristics were poured by evaluating LC patients consisted with those only in the advanced stage, some issues still remains unsolved. First, OS differences according to the presence of COPD in patients with squamous cell carcinoma or large-cell LC have not been analyzed due to the small number of patients in each of these conditions. Second, prognosis of patients treated with immune-checkpoint inhibitors has not been analyzed. Therefore, further studies including larger numbers of patients treated with homogeneous modalities are necessary to better elucidate the effects of COPD on LC patient prognosis.

\section{Conclusions}

Although no significant difference of OS according to the presence of COPD in patients with SCLC-ED and metastatic NSCLC was observed, adverse effects on metastatic NSCLC patients with moderate to very severe airflow limitation treated with chemotherapy were observed. Therefore, chemotherapy could be considered as a prior option in treatment of patients with SCLC-ED regardless of severity of airflow limitation and metastatic NSCLC classified GOLD 1.

\section{Acknowledgments}

None.

\section{Footnote}

Conflicts of Interest: The authors have no conflicts of interest to declare. 
Ethical Statement: The authors are accountable for all aspects of the work in ensuring that questions related to the accuracy or integrity of any part of the work are appropriately investigated and resolved. This study was carried out according to the principles in the 1964 Declaration of Helsinki, and an ethical committee approved the study protocol (2018GR0219).

\section{References}

1. Siegel RL, Miller KD, Jemal A. Cancer statistics, 2018. CA Cancer J Clin 2018;68:7-30.

2. Jian ZH, Huang JY, Ko PC, et al. Impact of coexisting pulmonary diseases on survival of patients with lung adenocarcinoma: a STROBE-compliant article. Medicine (Baltimore) 2015;94:e443.

3. Zhai R, Yu X, Shafer A, et al. The impact of coexisting COPD on survival of patients with early-stage non-small cell lung cancer undergoing surgical resection. Chest 2014;145:346-53.

4. Jeppesen SS, Hansen NG, Schytte T, et al. Comparison of survival of chronic obstructive pulmonary disease patients with or without a localized non-small cell lung cancer. Lung Cancer 2016;100:90-5.

5. Gullon JA, Suarez I, Medina A, et al. Role of emphysema and airway obstruction in prognosis of lung cancer. Lung Cancer 2011;71:182-5.

6. Lee SJ, Lee J, Park YS, et al. Impact of chronic obstructive pulmonary disease on the mortality of patients with nonsmall-cell lung cancer. J Thorac Oncol 2014;9:812-7.

7. Izquierdo JL, Resano P, El Hachem A, et al. Impact of COPD in patients with lung cancer and advanced disease treated with chemotherapy and/or tyrosine kinase inhibitors. Int J Chron Obstruct Pulmon Dis 2014;9:1053-8.

8. Omote N, Hashimoto N, Morise M, et al. Impact of mild to moderate COPD on feasibility and prognosis in nonsmall cell lung cancer patients who received chemotherapy. Int J Chron Obstruct Pulmon Dis 2017;12:3541-7.

9. Lim JU, Yeo CD, Rhee CK, et al. Overall survival of driver mutation-negative non-small cell lung cancer patients with COPD under chemotherapy compared to non-COPD non-small cell lung cancer patients. Int J Chron Obstruct Pulmon Dis 2018;13:2139-46.

10. Detterbeck FC, Boffa DJ, Kim AW, et al. The Eighth edition lung cancer stage classification. Chest 2017;151:193-203.

11. Gao YH, Guan WJ, Liu Q, et al. Impact of COPD and emphysema on survival of patients with lung cancer:
A meta-analysis of observational studies. Respirology 2016;21:269-79.

12. Reck M, Rodriguez-Abreu D, Robinson AG, et al. Pembrolizumab versus chemotherapy for PD-L1positive non-small-cell lung cancer. N Engl J Med 2016;375:1823-33.

13. Gandhi L, Rodriguez-Abreu D, Gadgeel S, et al. Pembrolizumab plus chemotherapy in metastatic nonsmall-cell lung cancer. N Engl J Med 2018;378:2078-92.

14. Borghaei H, Paz-Ares L, Horn L, et al. Nivolumab versus docetaxel in advanced nonsquamous non-small-cell lung cancer. N Engl J Med 2015;373:1627-39.

15. Brahmer J, Reckamp KL, Baas P, et al. Nivolumab versus docetaxel in advanced squamous-cell non-small-cell lung cancer. N Engl J Med 2015;373:123-35.

16. Masters GA, Temin S, Azzoli CG, et al. Systemic therapy for stage IV non-small-cell lung cancer : American Society of Clinical Oncology clinical practice guideline update. J Clin Oncol 2015;33:3488-515.

17. Cho O, Oh YT, Chun M, et al. Prognostic implication of FEV1/FVC ratio for limited-stage small cell lung cancer. J Thorac Dis 2018;10:1797-805.

18. Kang HS, Shin AY, Yeo CD, et al. A lower level of forced expiratory volume in one second predicts the poor prognosis of small cell lung cancer. J Thorac Dis 2018;10:2179-85.

19. Ju S, Lee HR, Kim JY, et al. Impact of coexistent chronic obstructive pulmonary disease on the survival of patients with small cell lung cancer receiving chemotherapy. Thorac Cancer 2018;9:1271-8.

20. The Korean Academy of Tuberculosis and Respiratory Diseases. 2016 Guideline of pulmonary function test. Seoul: The Korean Academy of Tuberculosis and Respiratory Diseases; 2016. [Nov 2016]. Available online: http://www.lungkorea.org/bbs/index.html? code=guide\&ca tegory $=\&$ gubun $=\&$ page $=1 \&$ number $=3487 \&$ mode $=v i e w \& \mathrm{k}$ eyfield $=\&$ key $=$

21. Patel AR, Patel AR, Singh S, et al. Global Initiative for Chronic Obstructive Lung Disease: The changes made. Cureus 2019;11:e4985.

22. Eisenhauer EA, Therasse P, Bogaerts J, et al. New response evaluation criteria in solid tumours: revised RECIST guideline (version 1.1). Eur J Cancer 2009;45:228-47.

23. Lim JU, Lee JH, Kim JS, et al. Comparison of World Health Organization and Asia-Pacific body mass index classifications in COPD patients. Int J Chron Obstruct Pulmon Dis 2017;12:2465-75.

24. Oken MM, Creech RH, Tormey DC, et al. Toxicity and 
response criteria of the Eastern Cooperative Oncology Group. Am J Clin Oncol 1982;5:649-55.

25. Semrau S, Klautke G, Fietkau R. Baseline cardiopulmonary function as an independent prognostic factor for survival of inoperable non-small-cell lung cancer after concurrent chemoradiotherapy: a single-center analysis of 161 cases. Int J Radiat Oncol Biol Phys 2011;79:96-104.

26. Videtic GM, Stitt LW, Ash RB, et al. Impaired diffusion capacity predicts for decreased treatment tolerance and survival in limited stage small cell lung cancer patients treated with concurrent chemoradiation. Lung Cancer 2004;43:159-66.

27. Brunelli A, Kim AW, Berger KI, et al. Physiologic evaluation of the patient with lung cancer being considered for resectional surgery: Diagnosis and management of lung cancer, 3rd ed: American College of Chest Physicians evidence-based clinical practice guidelines. Chest 2013;143:e166S-e190S.

28. van de Schans SA, Janssen-Heijnen ML, Biesma B, et al. COPD in cancer patients: higher prevalence in the elderly, a different treatment strategy in case of primary tumours above the diaphragm, and a worse overall survival in the elderly patient. Eur J Cancer 2007;43:2194-202.

29. Dong W, Du Y, Ma S. Impact of chemotherapy in the prognosis of non-small-cell lung cancer patients with severe to very severe COPD. Int J Chron Obstruct Pulmon Dis 2018;13:3805-12.

30. Bosken CH, Wei Q, Amos CI, et al. An analysis of DNA repair as a determinant of survival in patients with nonsmall-cell lung cancer. J Natl Cancer Inst 2002;94:1091-9.

31. Durham AL, Adcock IM. The relationship between COPD and lung cancer. Lung Cancer 2015;90:121-7.

32. Lee JH, Song EM, Sim YS, et al. Forced expiratory volume in one second as a prognostic factor in advanced non-small cell lung cancer. J Thorac Oncol 2011;6:305-9.

33. Wang $W$, Dou $S$, Dong $W$, et al. Impact of COPD on prognosis of lung cancer: from a perspective on disease heterogeneity. Int J Chron Obstruct Pulmon Dis 2018;13:3767-76.

Cite this article as: Lee SY, Choi YJ, Seo JH, Lee SY, Kim JS, Kang EJ. Pulmonary function is implicated in the prognosis of metastatic non-small cell lung cancer but not in extended disease small cell lung cancer. J Thorac Dis 2019;11(11):45624572. doi: $10.21037 /$ jtd.2019.10.77 